\title{
Iterative Near Maximum-Likelihood Sequence Detection for MIMO Optical Wireless Systems
}

\author{
Nestor D. Chatzidiamantis, Student Member, IEEE, Murat Uysal, Senior Member, IEEE, \\ Theodoros A. Tsiftsis, Member, IEEE, and George K. Karagiannidis, Senior Member, IEEE
}

\begin{abstract}
A major performance-limiting factor in terrestrial optical wireless (OW) systems is turbulence-induced fading. Exploiting the additional degrees of freedom in the spatial dimension, multiple laser transmitters combined with multiple receive apertures provide an effective solution for fading mitigation. Although multiple-input multiple-output (MIMO) OW systems have been extensively studied in recent years, most of these works are mainly limited to symbol-by-symbol decoding. MLSD exploits the temporal correlation of turbulence-induced fading and promises further performance gains. In this paper, we investigate MLSD for intensity-modulation/direct-detection MIMO OW systems over log-normal atmospheric turbulence channels. Even with a low-order modulation scheme such as OOK, which is typically used in OW systems, the complexity of MLSD might be prohibitive. We therefore present an iterative sequence detector based on the expectation-maximization (EM) algorithm. The complexity of the proposed algorithm is considerably less than a direct evaluation of the log-likelihood function, and it is independent of the channel's fading statistics. The Monte Carlo simulation results demonstrate that the EM-based algorithm outperforms the symbol-by-symbol decoder and achieves a performance, which lies within $0.3 \mathrm{~dB}$ of that of the optimal MLSD.
\end{abstract}

Index Terms-Atmospheric turbulence, expectation-maximization (EM) algorithm, free-space optical communication, MLSD, maximum-likelihood (ML) symbol-by-symbol detection, spatial diversity.

\section{INTRODUCTION}

W IRELESS technology is traditionally associated with RF transmission although transmission via carriers in the other parts of electromagnetic spectrum might be more advantageous for various applications [1]. Operating at unlicensed optical wavelengths, terrestrial optical wireless (OW) systems offer the potential of broadband communication capacity that no other wireless transmission. As a cost-effective alternative and/or complement to RF counterparts, OW systems present an

\footnotetext{
Manuscript received November 18, 2008; revised September 02, 2009, November 18, 2009. First publishedDecember 22, 2009; current version publishedMarch 05, 2010. This paper was presented in part at the IEEE International Conference on Communications, Dresden, Germany, 2009.

N. D. Chatzidiamantis and G. K. Karagiannidis are with the Wireless Communications Systems Group, Department of Electrical and Computer Engineering, Aristotle University of Thessaloniki, GR-54124 Thessaloniki, Greece (e-mail: nestoras@ auth.gr; geokarag@ auth.gr).

M. Uysal is with the School of Engineering, Özyeğin University, 34662 Istanbul, Turkey (e-mail: murat.uysal@ozyegin.edu.tr).

T. A. Tsiftsis is with the Department of Electrical Engineering, Technological Educational Institute of Lamia, 35100 Lamia, Greece (e-mail: tsiftsis@teilam. gr).

Digital Object Identifier 10.1109/JLT.2009.2038724
}

attractive remedy for the "last mile" problem, i.e., to provide broadband wireless extensions to Internet backbones bridging the gap between the end user and the infrastructure already in place [2]. With their broadband capacity, they can be further deployed for other high data rate applications such as metropolitan area network extensions, enterprise/local area network connectivity, fiber backup, and backhaul for wireless cellular networks. Due to their features such as flexibility, rapid deployment time, high security, and immunity to RF interference, OW systems are also appealing as a redundant link for relief efforts, disaster recovery, and military applications.

Despite their significant advantages, OW systems unfortunately have some shortcomings, which need to be addressed to make possible their widespread deployment. The major limitation in the performance of OW systems is their high vulnerability to adverse atmospheric conditions. Even in a clear sky, due to inhomogeneities in temperature and pressure changes, the refractive index of the atmosphere varies and results in atmospheric turbulence. This causes rapid fluctuations at the received signal, known as turbulence-induced fading. Such fluctuations lead to an increase in the error rate performance, thereby severely affecting the reliability of OW link. Over the last years, several fading mitigation techniques have been proposed for deployment in OW links to combat the degrading effects of atmospheric turbulence. One of such techniques is error control coding (ECC), which has been investigated in [3] and [4]. ECC in conjunction with interleaving is known in the RF literature to provide an effective time-diversity solution for rapidly varying fading channels. In the OW systems, data rates can typically be of the order of gigabits per second. With a correlation time of the order of $10^{-3}$ to $10^{-2} \mathrm{~s}$, OW channels exhibit slow fading; therefore the practical use of ECC in OW links is rather limited due to the required large-size interleavers to achieve the promising coding gains theoretically available.

An effective solution for fading mitigation is spatial diversity techniques, which involve the deployment of multiple transmit/ receive apertures. Introducing additional degrees of freedom in the spatial dimension, multiple-input multiple-output (MIMO) OW systems promise significant performance gains. The performance of MIMO OW systems has been extensively studied by various authors assuming different type of fading channels, noise statistics, and modulation type [5]-[8]. These works however are mainly limited to the assumption of symbol-by-symbol decoding. It is known that MLSD exploits the temporal characteristics of the fading channels and outperforms the conventional symbol-by-symbol maximum-likelihood (ML) decoder. It proves to be particularly effective for slowly varying fading 
or quasi-static fading channels as the turbulence-induced fading turns out to be. MLSD in the context of OW communication has been first investigated by Zhu and Kahn in [9], [10], and [11], for a single-input single-output (SISO) scenario. However, MLSD involves the computation of complicated multidimensional integrals and therefore suffers from high complexity. Furthermore, it requires knowledge of the channel's fading statistics. To address the complexity issue, suboptimal, yet of low complexity, MLSD metrics have been proposed in [12] and [13] for different detection models; still, these metrics require knowledge of the channel's statistics. Other detection methods such as pilot-symbol assisted modulation (PSAM) or blind detection, which both do not require knowledge of the channel's fading statistics have been also applied to SISO OW systems in [11] and [14], respectively.

In this study, we aim to investigate MLSD solutions for MIMO OW systems as a powerful combination for fading mitigation in atmospheric turbulence channels. Since MLSD in a MIMO scenario suffers from excessive complexity and is infeasible for most practical purposes, we propose an iterative ML sequence detector based on the expectation-maximization (EM) algorithm. The EM algorithm has been originally proposed by Dempster et al. [15] in the statistics literature as a general procedure for iterative ML estimation. Since then, it has been widely applied to a variety of communication problems [16]-[18]. This algorithm is particularly useful when the estimation problem is made difficult by the absence of certain information, e.g., absence of full channel state information in a data detection problem. In this paper, we propose EM algorithm as a low-complexity solution for MLSD in the MIMO OW system under consideration. As benchmarks, we also investigate the performance of symbol-by-symbol ML detector, blind detector of [14], PSAM, and MLSD in a MIMO scenario. The proposed detector outperforms symbol-by-symbol ML detector, blind detector, and PSAM methods. Moreover, its performance lies within $0.3 \mathrm{~dB}$ of the MLSD at a much lower complexity and converges to MLSD as the strength of turbulence increases.

The remainder of the paper is organized as follows. In Section II, we describe the MIMO OW system and the turbulence-induced fading model. In Section III, we present symbol-by-symbol and sequence-based ML detectors, which will be used as benchmarks for system performance and complexity, and introduce the EM algorithm for the MIMO OW system under consideration. In Section IV, we present Monte Carlo simulation results to demonstrate the error rate performance of the proposed detection technique and compare it with benchmarking schemes. Finally, in Section V, we provide our concluding remarks.

Notations: $\mathbf{x}^{T}$ denotes the transpose of the matrix $\mathbf{x} ; \mathrm{E}[\cdot]$ denotes statistical expectation; $\|\mathbf{x}\|$ denotes the norm of the vector $\mathbf{x} ; N\left(\mu, \sigma^{2}\right)$ denotes Gaussian distribution with mean $\mu$ and variance $\sigma^{2}$.

\section{System AND CHANNEL MODEL}

We consider a MIMO OW link with $N$ transmit and $M$ receive apertures. At the transmitter, data blocks of length $L$ are modulated using OOK and transmitted through the $N$ apertures, using repetition coding; an efficient transmission scheme for MIMO OW links [19].

We assume operation in the high SNR regime, where the shot noise caused by ambient light is dominant and therefore Gaussian noise model is used as a good approximation of the Poisson photon counting detection model [9]. Furthermore, a large field of view is considered for each receiver, indicating that multiple transmitters are simultaneously observed by each receiver. This actually leads to the collection of larger amount of background radiation, which further justifies the use of Gaussian noise model.

Let $\mathbf{s}=\left(s^{(1)} s^{(2)} \ldots s^{(L)}\right)^{T}$ be the $L \times 1$ column vector containing the $L$ modulated symbols within a data frame. The received signal at the $m$ th receive aperture is given as

$$
r_{m}^{(l)}=s^{(l)} \eta \sum_{n=1}^{N} I_{n m}^{(l)}+v_{m}, \quad m=1, \ldots M, l=1, \ldots L
$$

where $s^{(l)} \in\{0,1\}$ is the transmitted information bit, $\eta$ is the optical-to-electrical conversion coefficient, and $v_{m}$ is additive white Gaussian noise with zero mean and variance $\sigma_{v}^{2}=N_{o} / 2$. The fading coefficient, which models the atmospheric turbulence in the optical channel between the $n$th transmit aperture and the $m$ th receive aperture during the $l$ th symbol interval, is given by

$$
I_{n m}^{(l)}=I_{o} \exp \left(2 x_{n m}^{(l)}\right)
$$

where $I_{o}$ is the signal light intensity without turbulence and $x_{n m}^{(l)}$ are identically distributed normal random variables with mean $\mu_{x}$ and variance $\sigma_{x}^{2}$, i.e., $f_{x}\left(x_{n m}^{(l)}\right)=N\left(\mu_{x}, \sigma_{x}^{2}\right)$. Therefore, $I_{n m}^{(l)}$ follows a log-normal distribution with probability density function given by

$$
f\left(I_{n m}^{(l)}\right)=\frac{1}{2 I_{n m}^{(l)}} \frac{1}{\sqrt{2 \pi \sigma_{x}^{2}}} \exp \left(-\frac{\left(\ln \left(\frac{I_{n m}^{(l)}}{I_{o}}\right)-2 \mu_{x}\right)}{8 \sigma_{x}^{2}}\right) .
$$

To ensure that the fading does not attenuate or amplify the average power, we normalize the fading coefficients such that $\mathrm{E}\left[\left|\left(I_{n m}^{(l)}\right) /\left(I_{o}\right)\right|\right]=1$. Doing so requires the choice of $\mu_{x}=-\sigma_{x}^{2}$ [8]. The variances of log-amplitude fluctuation of plane and spherical waves can be found in [20].

Atmospheric turbulence results in a very slowly varying fading in OW systems. For the signaling rates of interest, ranging from hundreds to thousands of Mbps [21], the fading coefficient can be considered as constant over hundred of thousand or millions of consecutive symbols, since the coherence time of the channel is about 1-100 ms [5]. Hence, it is assumed that $I_{n m}^{(l)}$ remains constant over the symbols of a frame and therefore we drop the time index $l$, i.e.,

$$
I_{n m}^{(l)}=I_{n m}, \quad l=1, \ldots L
$$

and (1) can be written in a vector form as

$$
\mathbf{r}_{m}=\mathbf{s} \eta \sum_{n=1}^{N} I_{n m}+v_{m}, \quad m=1, \ldots M
$$


where $\mathbf{r}_{m}=\left(\begin{array}{llll}r_{m}^{(1)} & r_{m}^{(2)} & \ldots & r_{m}^{(L)}\end{array}\right)^{T}$ and $v_{m}$ is a $L \times 1$ column vector containing noise samples. Moreover, it is assumed that the distances between the transmitter and receiver apertures are large compared to the correlation length of intensity fluctuations. This assumption is realistic for many OW systems as it is described in [5]. Hence, the underlying channels can be considered as independent in space.

At the receiver, we assume equal gain combining, whose performance is very close to maximal ratio combining[8] (e.g., within $0.5 \mathrm{~dB}$ for $N=2,3$ apertures and $\sigma_{x}=0.3$ ). Thus, after combining the received signals of the $M$ apertures, the output of the receiver can be written in matrix form as

$$
\mathbf{r}=\frac{1}{N M} \sum_{m=1}^{M} \mathbf{r}_{m}=\frac{\eta}{N M} \mathbf{s} \sum_{m=1}^{M} \sum_{n=1}^{N} I_{n m}+\mathbf{n}
$$

where $\mathbf{n}$ is a $L \times 1$ column vector, which contains noise samples. Note that a scaling factor $(1) /(N M)$ appears in (6). The factor $(1 / N)$ is included in order to ensure that the total transmit power is the same with that of a system with no transmit diversity. The factor $(1 / M)$, on the other hand, ensures that the sum of the $M$ receive aperture areas is the same with the aperture area of a system with no receive diversity.

\section{ML DETECTION TECHNIQUES FOR MIMO OW SySTEMS}

In this section, we present detection techniques, which are based on the ML criterion and can be employed when instantaneous channel state information (CSI) is not available at the receiver.

\section{A. ML Symbol-by-Symbol Detection}

The ML symbol-by-symbol detector chooses the symbol $\hat{s}$ based on the rule [22]

$$
\hat{s}=\arg \max _{s} p(r \mid s)
$$

where $p(r \mid s)$ is the conditional probability of the received signal $r$ when $s$ is transmitted. The likelihood function for SISO OW systems is given by [9, eq. (30)]. For the MIMO OW system under consideration, the ML decision rule takes the form, as shown in (8), at the bottom of this page, where $\mathbf{x}=\left\{x_{n m}\right\}$ and, since it is assumed that the intensity fluctuations are independent in space, $f_{\mathbf{x}}(\mathbf{x})=\prod_{m=1}^{M} \prod_{n=1}^{N} f_{x}\left(x_{n m}\right)$. An efficient computation of (8) can be performed using the sum of log-normal approximation and Gauss-Hermite quadrature formula as discussed in [8].

\section{B. ML Sequence Detection}

This detection scheme exploits the temporal correlation of turbulence-induced fading over consecutive transmitted symbols. MLSD is based on the assumption that the receiver has knowledge of the marginal joint distribution of the intensity fluctuations, but not of their instantaneous state. It was first investigated in [9] for OW communications, assuming a SISO scenario. For the MIMO OW system with OOK, the MLSD computes the likelihood ratio of each of the $2^{L}$ possible sequences and chooses according to the decision rule as given by (9) at the bottom of this page, where $\mathbf{r}=\left[r_{1}, r_{2}, \ldots, r_{L}\right]$ represents the received data.

A major drawback of MLSD is its computational complexity, since it requires the computation of the $M N$-dimensional integral of (9) for each of the $2^{L}$ possible sequences. A suboptimal low-complexity implementation has been proposed in [10], adopting Markov model; it still requires the numerical integrations to be performed in each branch metric in the trellis search. In [12] and [13], another efficient low-complexity implementation is proposed, which uses analytically tractable decision metrics and searches over a subset of all possible sequences; however, in that case, there is a difficulty in calculating the parameters of the low-complexity decision metrics and knowledge of the channel's statistics is required.

\section{EM-Based Sequence Detection}

In this section, we propose an EM-based sequence detector as a low-complexity alternative to the original MLSD. This scheme is a two-step iterative procedure, which estimates both the transmitted sequence and the channel state based on its previous estimates.

A general description of the EM algorithm can be found in [15]. For its application in fading channels and MIMO RF communication systems, the reader is urged to read [16]-[18]. With the available data only, i.e., the "incomplete data set" according

$$
\hat{s}=\arg \max _{s}\left[\int_{\mathbf{x}} f_{\mathbf{x}}(\mathbf{x}) \exp \left(-\frac{\left(r-\frac{\eta}{N M} \sum_{m=1}^{M} \sum_{n=1}^{N} e^{2 x_{n m}}\right)^{2}-r^{2}}{N_{o}}\right) d \mathbf{x}\right]
$$

$$
\hat{\mathbf{s}}=\arg \max _{\mathbf{s}} p(\mathbf{r} \mid \mathbf{s})=\arg \max _{\mathbf{s}} \int_{\mathbf{x}} f_{\mathbf{x}}(\mathbf{x}) \exp \left[-\sum_{l=1}^{L}-\frac{\left(r^{(l)}-s^{(l)} \frac{\eta}{N M} \sum_{m=1}^{M} \sum_{n=1}^{N} e^{2 x_{n m}}\right)^{2}}{N_{o}}\right] d \mathbf{x}
$$


to the EM terminology, finding the ML estimates might be computationally intensive as in our case. Including a proper selection of another data set resulting in so-called "complete data set," it might be made easier to compute the ML estimates. Since not all the elements of the complete data set are known, the EM algorithm makes use of the log-likelihood function for the complete data in a two-step iterative procedure, iterating between expectation step ( $E$-step) and maximization step ( $M$-step).

In our study, the received sequence $\mathbf{r}$ is the incomplete data set. Let the complete data be $\mathbf{y}=\left(\mathbf{r},\left\{I_{n m}\right\}\right)$. After using the likelihood function for MIMO OW systems, as in (9), the $\log$-likelihood function for the complete data in vector form is obtained by

$$
l\left(\mathbf{r} \mid \mathbf{s},\left\{I_{n m}\right\}\right)=\frac{1}{N_{o}}\left\|\mathbf{r}-\mathbf{s} \frac{\eta}{N M} \sum_{m=1}^{M} \sum_{n=1}^{N} I_{n m}\right\|^{2} .
$$

After dropping some unnecessary terms, (10) reduces to

$$
\begin{aligned}
l\left(\mathbf{r} \mid \mathbf{s},\left\{I_{n m}\right\}\right)=\mathbf{r}^{T} \mathbf{s} \frac{\eta}{N M} & \sum_{m=1}^{M} \sum_{n=1}^{N} I_{n m} \\
& -\frac{1}{2}\left\|\mathbf{s} \frac{\eta}{N M} \sum_{m=1}^{M} \sum_{n=1}^{N} I_{n m}\right\|^{2}
\end{aligned}
$$

or equivalently

$$
\begin{aligned}
l\left(\mathbf{r} \mid \mathbf{s},\left\{I_{n m}\right\}\right)=\sum_{l=1}^{L} & r^{(l)} s^{(l)} \frac{\eta}{N M} \sum_{m=1}^{M} \sum_{n=1}^{N} I_{n m} \\
& -\frac{1}{2} \sum_{l=1}^{L}\left(s^{(l)} \frac{\eta}{N M} \sum_{m=1}^{M} \sum_{n=1}^{N} I_{n m}\right)^{2}
\end{aligned}
$$

At the $E$-step of the $k$ th iteration of the algorithm, the log-likelihood function for the complete data is calculated, conditioned on the received sequence $\mathbf{r}$ and the $k$ th estimate of the transmitted sequence $\mathbf{s}^{k}$. Hence, based on (11) or (12) we obtain

$$
Q\left(\mathbf{s} \mid \mathbf{s}^{k}\right)=\mathbf{r}^{T} \mathbf{s} I^{k}-\frac{1}{2}\left\|\mathbf{s} I^{k}\right\|^{2}
$$

where

$$
I^{k}=\mathrm{E}\left[\frac{\eta}{N M} \sum_{m=1}^{M} \sum_{n=1}^{N} I_{n m} \mid \mathbf{s}^{k}, \mathbf{r}\right]=\frac{1}{L_{\mathrm{on}}^{k}} \mathbf{r}^{T} \mathbf{s}^{k}
$$

and $L_{\mathrm{on}}^{k}$ is the number of the bits of the $k$ th estimate of the transmitted sequence $\mathbf{s}^{k}$ that correspond to the ON-state.

A better estimate of the transmitted sequence, $\mathbf{s}^{k+1}$, is obtained at the second step of the $k$ th iteration of the algorithm, the $M$-step. $M$-step performs the maximization of (13), which yields

$$
\mathbf{s}^{k+1}=\arg \max _{\mathbf{s}}\left(\mathbf{r}^{T} \mathbf{s} I^{k}-\frac{1}{2}\left\|\mathbf{s} I^{k}\right\|^{2}\right)
$$

Since the transmitted data bits are randomly chosen, i.e., no coding scheme is employed, maximizing (15) over the $L$-bit sequences is equivalent to making symbol-by-symbol decisions on each bit. In other words, if $\mathbf{s}^{k+1}$ is the sequence that maximizes (15), its components are easily obtained through

$$
\begin{array}{r}
\left(s^{k+1}\right)^{(l)}=\arg \max _{s^{(l)}}\left(r^{(l)} s^{(l)} I^{k}-\frac{1}{2}\left\|s^{(l)} I^{k}\right\|^{2}\right) \\
l=1, \ldots, L .
\end{array}
$$

Unlike MLSD, this detection scheme does not require knowledge of the marginal joint distribution of the intensity fluctuations at the receiver. Moreover, it avoids the numerical calculation of (9), which can be very complex for high SNRs. However, it needs an initial estimate of the channel state $I^{0}$ in order to initialize the algorithm.

The insertion of pilot channel estimation bits at each data frame at the transmitter is one possible way to obtain an initial channel estimate. Assume that pilot symbols are inserted into the modulated data blocks at every $J$ symbols. In making decisions, the receiver makes observation on data vector of $F$ subblocks, each with a length of $J$ (i.e., $L=F J$ symbols), for some integer $F$. Of the $J$ symbols in the subblock, the first is a pilot symbol, which is in the ON-state. The initial estimate of the channel's state is obtained by taking the average of the pilot symbols, i.e.,

$$
I^{0}=\frac{1}{F} \sum_{i=0}^{F-1} r^{(1+i J)} .
$$

The implementation of the blind detection method of [14] is another possible way to initialize EM algorithm. This method avoids the use of pilot symbols and obtains an initial estimate of the transmitted sequence $\mathbf{s}^{0}$ by performing symbol-by-symbol detection, according to decision rule

$$
\begin{aligned}
\left(s^{0}\right)^{(l)} & =1 \\
\left(s^{0}\right)^{(l)} & =0
\end{aligned} \tau_{\text {blind }}, \quad l=1, \ldots L
$$

where

$$
\tau_{\text {blind }}=\frac{1}{L} \sum_{l=1}^{L} r^{(l)} .
$$

Hence, by using (14), the channel estimate that is needed in order to initialize the EM algorithm can be obtained.

It should be noted that the quality of the initial channel estimates and/or the length of the data frames determines the convergence of the performance of the EM-based receiver to that of the perfect CSI receiver [16].

\section{Simulations Results AND Discussion}

In this section, we present simulation results for the bit-error-rate (BER) performance of the MIMO OW communication system with EM-based receiver for various numbers of transmit/receive apertures. We further compare its performance with other competing detection techniques.

In Fig. 1, we consider a multiple-transmit single-output (MISO) OW system with $N=3$ transmit apertures and a 


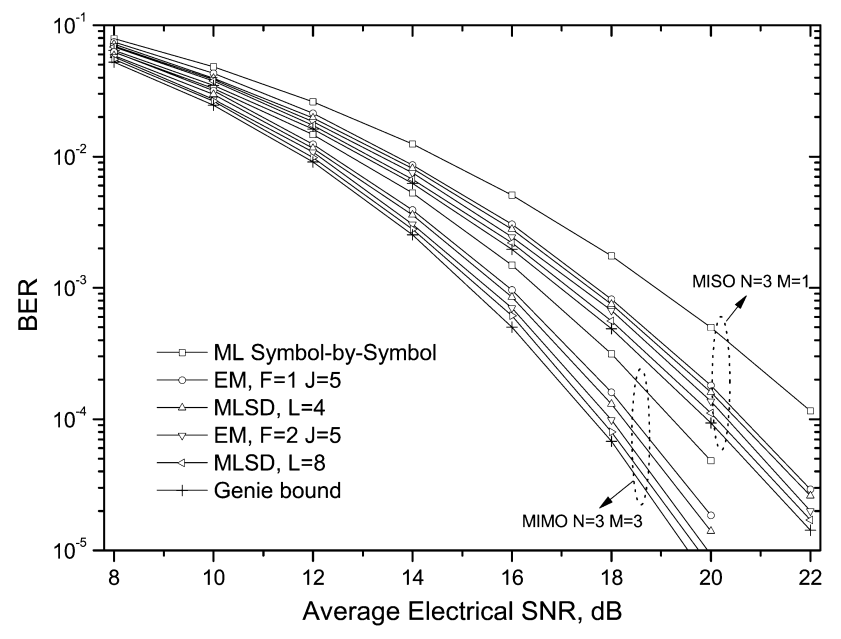

Fig. 1. Comparison of symbol detection techniques for MIMO and MISO OW systems when $\sigma_{x}=0.3$.

MIMO system with $N=3$ transmit and $M=3$ receive apertures over the log-normal turbulence channel with $\sigma_{x}=0.3$. The performance of the proposed EM algorithm that uses pilot symbols for initialization, along with ML symbol-by-symbol decoder and MLSD is illustrated. We assume two iterations in the implementation of EM algorithm since further iterations results in a negligible performance improvement. Among the three competing schemes, symbol-by-symbol detection scheme performs worst. This is expected, since this detector does not take advantage of the fading correlation between successive symbols that exists in turbulence channels. Hence, the use of sequence detection techniques, such as MLSD or EM is fully justified. As an ultimate benchmark, we also include the performance of genie receiver, which assumes perfect CSI and acts as a lower bound on the performance of other detection types. MLSD performs very close to the genie bound and slightly outperforms the EM detector. Specifically, there is an SNR improvement of approximately $0.3 \mathrm{~dB}$ at BER $=10^{-4}$ between MLSD of $L=4$ and EM of $(F=1, J=5)$ or between MLSD of $L=8$ and EM of $(F=2, J=5)$ in both MIMO OW systems. However, it should be noted that the complexity of the MLSD implementation is much higher than that of EM algorithm, i.e., MLSD requires the calculation of an $N M$-dimensional integral for each of the $(L+1)$ possible sequences ${ }^{1}$, which is very difficult especially for the SNR regime under consideration. That is also the same reason why we restricted ourselves to small values of frame lengths. Otherwise, simulation of MLSD would not be possible for comparison purposes.

Fig. 2 depicts the performance of the MIMO OW system under consideration for the same number of transmit and receive apertures, but for stronger turbulence conditions with $\sigma_{x}=$ 0.4 . It is observed that the proposed EM-based detection technique still performs very close to the optimal MLSD, despite the increase of the turbulence strength. Specifically, the SNR improvement in this case is approximately $0.1 \mathrm{~dB}$ for the same

\footnotetext{
${ }^{1}$ In simulations, the low-complexity search algorithm, presented in [12] and [13], was employed, which evaluates the MLSD metric for $(L+1)$ out of the $2^{L}$ possible sequences.
}

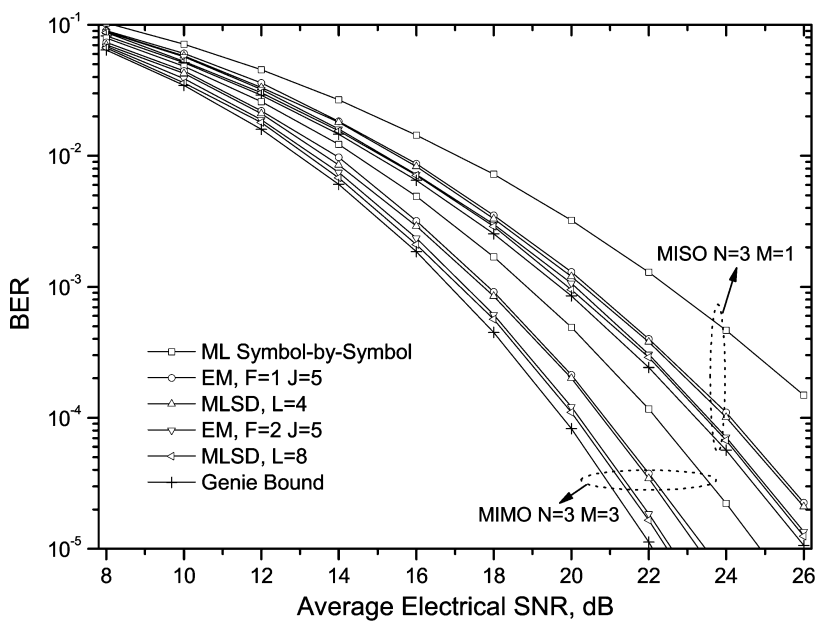

Fig. 2. Comparison of symbol detection techniques for MIMO and MISO OW systems when $\sigma_{x}=0.4$.

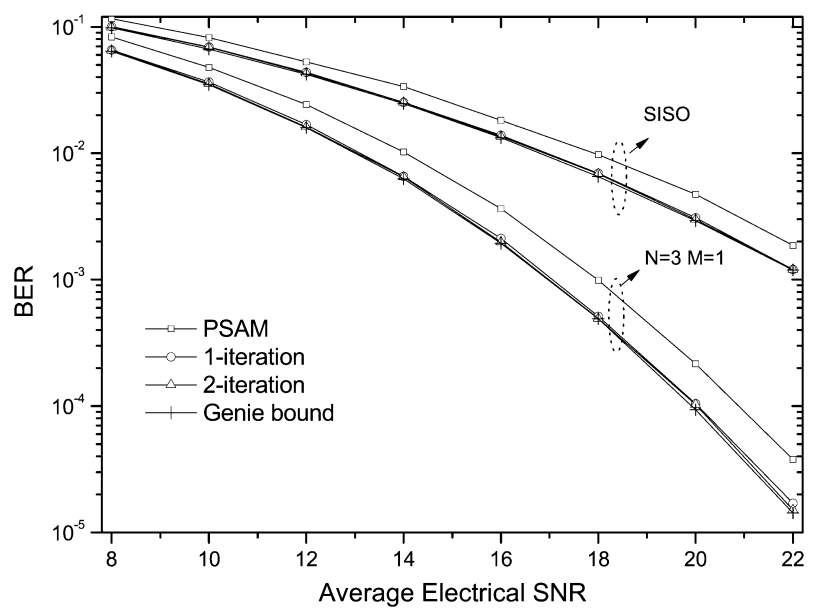

Fig. 3. Performance of EM-based receiver for $F=1$ and $L=100$ and comparison with PSAM.

combinations of $L, F$, and $J$ in both MIMO OW systems. This convergence of the EM-based detection to the MLSD is expected, since the proposed algorithm depends mostly on the frame length, in contrast with MLSD, which has a strong dependency from the channel's statistics.

In Fig. 3, we investigate the performance of EM algorithm for a large frame length, which would be prohibitive for MLSD implementation. Furthermore, large frame lengths make the loss due to pilot insertion negligible. Specifically, we consider the SISO and the MISO OW systems with $L=100$ over the lognormal turbulence-induced fading model with $\sigma_{x}=0.3$, assuming subblock length of $J=100$ and pilot symbol number $F=1$. The performance of proposed EM algorithm is illustrated assuming one and two iterations. It is observed that the performance of EM detector lies within $0.1 \mathrm{~dB}$ of the genie bound for $F=1$ and $J=100$. As another benchmark, we also include the performance of PSAM with variable threshold [11], i.e., the decision rule for the detection of each symbol in the frame, is defined entirely by the channel estimate of the pilot 


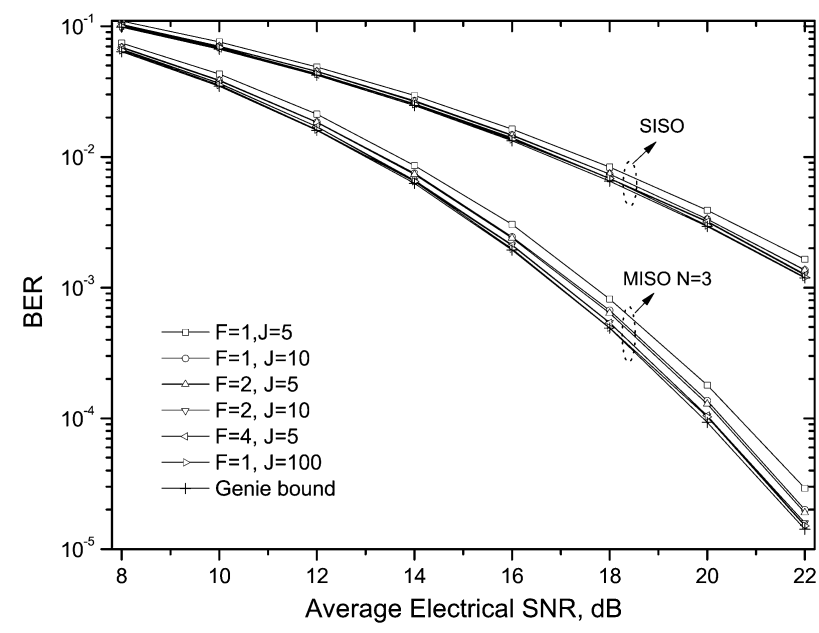

Fig. 4. Performance of EM-based receiver for various number of pilot symbols $F$ and data-block lengths $L(L=F J)$.

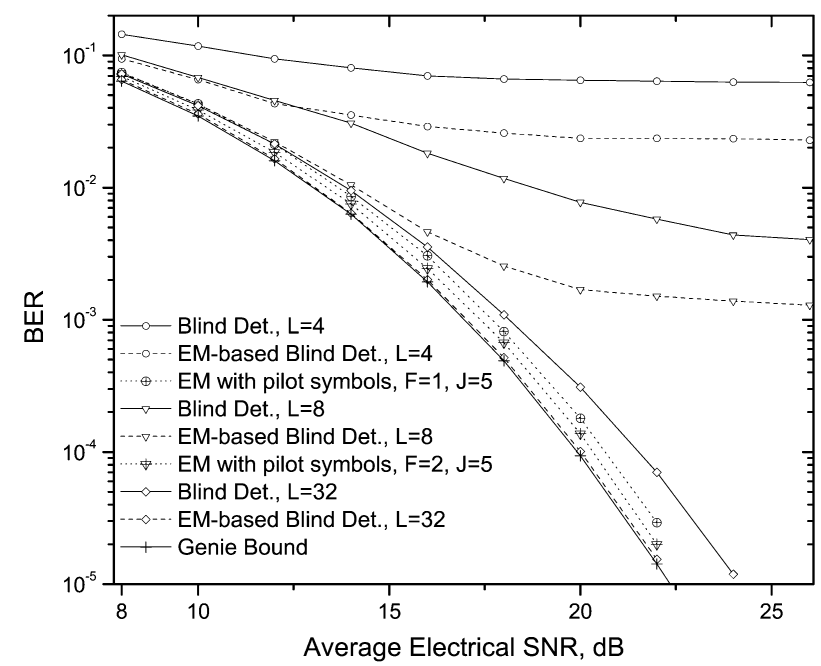

Fig. 5. Performance of EM-based blind detection scheme along with the blind detection without EM and the pilot symbol assisted EM.

symbols. The EM algorithm outperforms PSAM by approximately $1 \mathrm{~dB}$.

Fig. 4 depicts the performance of the EM-based receiver, when $\sigma_{x}=0.3$, for different frame lengths, assuming various combinations of $F$ and $J$ values. Specifically, we assume the combinations of $F=1$ and $J=5, F=1$ and $J=10, F=2$ and $J=5, F=2$ and $J=10, F=4$ and $J=5$, and finally $F=1$ and $J=100$. It is observed that the increase of the length and/or the number of pilot symbols helps EM-based receiver to perform closer to the genie bound. It should be noted that the complexity of the algorithm does not increase significantly as the length of data blocks increases, because EM implementation does not involve the computation of $N M$-dimensional integrals and, unlike MLSD, the increase of frame length increases only the number of the symbol-by-symbol decisions performed at the $M$-step of the algorithm.

Finally, in Fig. 5, we investigate the performance of EM-algorithm that uses the blind detection method of [14] for algorithm initialization, when $\sigma_{x}=0.3$ and $N=3$ transmit and
$M=1$ receive apertures are assumed. It is obvious from the figure that the application of the EM algorithm significantly increases the system performance compared to the blind detection scheme of [14]. Specifically, there is an improvement of $2 \mathrm{dBs}$ (at BER $=10^{-4}$ ) when $L=32$, while the error floors are reduced when smaller sequence lengths are considered $(L=4$ and $L=8$ ). Furthermore, when compared with the pilot-symbol assisted EM-based receiver, it is observed that the latter has better performance for the same frame length (no error floors are observed). However, as the frame length increases, the performance of the EM-based blind detection is improved, since the quality of the initial estimate is improved, and converges to the genie bound. Hence, both EM implementations can be employed with the same success for large frame lengths, i.e., large number of consecutive symbols where the fading coefficient remains constant, which is easily justified for the slowly varying atmospheric turbulence channels.

\section{CONCLUSION}

We have investigated sequence detection techniques for MIMO OW systems in the presence of turbulence-induced fading. Sequence detection exploits the temporal correlation of OW channel and promises significant performance gains over the symbol-by-symbol decoding. However, optimal ML sequence detector is infeasible for most practical purposes due to its high complexity. In our study, we have proposed a low-complexity sequence detector based on the EM algorithm. EM algorithm provides an iterative ML solution, which avoids the calculation of the difficult multidimensional integrals involved in the MLSD. Moreover, since it does not take into consideration the channel's fading statistics, it can be applied in different turbulence conditions, regardless their strength. Our simulation results demonstrate that the proposed EM solution performs very close to that of the MLSD. Specifically, it has been observed that the performance of EM lies within $0.3 \mathrm{~dB}$ when $\sigma_{x}=0.3$ or within $0.1 \mathrm{~dB}$ when $\sigma_{x}=0.4$ (at $\mathrm{BER}=10^{-4}$ ) of that of MLSD for the MIMO OW system under consideration.

\section{REFERENCES}

[1] L. Andrews, R. L. Philips, and C. Y. Hopen, Laser Beam Scintillation With Applications. Bellingham, WA: SPIE Press, 2001.

[2] D. Kedar and S. Arnon, "Urban optical wireless communications networks: The main challenges and possible solutions," IEEE Commun. Mag., vol. 42, no. 5, pp. 2-7, Feb. 2003.

[3] X. Zhu and J. M. Kahn, "Performance bounds for coded free-space optical communications through atmospheric turbulence channels," IEEE Trans. Commun., vol. 51, no. 8, pp. 1233-1239, Aug. 2003.

[4] M. Uysal, S. M. Navidpour, and J. Li, "Error rate performance of coded free-space optical links over strong turbulence channels," IEEE Commun. Lett., vol. 8, no. 10, pp. 635-637, Oct. 2004.

[5] E. Lee and V. Chan, "Part 1: Optical communication over the clear turbulent atmospheric channel using diversity," IEEE J. Sel. Areas Commun., vol. 22, no. 9, pp. 1896-1906, Nov. 2004

[6] S. G. Wilson, M. Brandt-Pearce, C. Qianling, and M. Baedke, "Optical repetition MIMO transmission with multipulse PPM," IEEE J. Sel. Areas Commun., vol. 23, no. 9, pp. 1901-1910, Jan. 2005.

[7] T. A. Tsiftsis, H. G. Sandalidis, G. K. Karagiannidis, and M. Uysal, "Optical wireless links with spatial diversity over strong atmospheric turbulence channels," IEEE Trans. Wireless Commun., vol. 8, no. 2, pp. 951-957, Feb. 2009. 
[8] S. M. Navidpour and M. Uysal, "BER performance of free-space optical transmission with spatial diversity," IEEE Trans. Wireless Commun., vol. 6, no. 8, pp. 2813-2819, Aug. 2007.

[9] X. Zhu and J. M. Kahn, "Free-space optical communication through atmospheric turbulence channels," IEEE Trans. Commun., vol. 50, no. 8, pp. 1293-1300, Aug. 2002.

[10] X. Zhu and J. M. Kahn, "Markov chain model in maximum-likelihood sequence detection for free-space optical communication through atmospheric turbulence channels," IEEE Trans. Commun., vol. 51, no. 3, pp. 509-516, Mar. 2003.

[11] X. Zhu and J. M. Kahn, "Pilot-symbol assisted modulation for correlated turbulent free-space optical channels," presented at the SPIE Int. Symp. Opt. Sci. Technol., San Diego, CA, 2001.

[12] M. L. B. Riediger, R. Schober, and L. Lampe, "Fast multiple-symbol detection for free-space optical communications," IEEE Trans. Commun., vol. 57, no. 4, pp. 1119-1128, Apr. 2009.

[13] M. L. B. Riediger, R. Schober, and L. Lampe, "Fast multiple-symbol detection for photon-counting MIMO free-space optical communications," IEEE Trans. Wireless Commun., vol. 7, no. 12, pp. 5369-5379, Dec. 2008.

[14] M. L. B. Riediger, R. Schober, and L. Lampe, "Blind detection of on-off keying for free-space optical communications," in Proc. CCECE/CCGEI, Niagara Falls, Canada, 2008, pp. 1361-1364.

[15] A. Dempster, N. M. Laird, and D. B. Rubin, "Maximum-likelihood from incomplete data via the EM algorithm," J. R. Statist. Soc., vol. 39, pp. 1-17, 1977.

[16] C. N. Georghiades and J. C. Han, "Sequence estimation in the presence of random parameters via the EM algorithm," IEEE Trans. Commun., vol. 45, no. 3, pp. 300-308, Mar. 1997.

[17] C. Cozzo and B. L. Hughes, "Joint channel estimation and data detection in space-time communications," IEEE Trans. Commun., vol. 51, no. 8, pp. 1266-1270, Aug. 2003.

[18] Y. Li, C. N. Georghiades, and G. Huang, "Iterative maximum-likelihood sequence estimation for space-time coded systems," IEEE Trans. Commun., vol. 49, no. 6, pp. 948-951, Jun. 2001.

[19] M. Safari and M. Uysal, "Do we really need OSTBCs for free-space optical communication with direct detection?," IEEE Trans. Wireless Commun., vol. 7, no. 11, pp. 4445-4448, Nov. 2008.

[20] S. Karp, R. Gagliardi, S. E. Moran, and L. B. Stotts, Optical Channels. New York: Plenum, 1988.

[21] D. J. T. Heatley, D. R. Wisely, I. Neild, and P. Cochrane, "Optical wireless: The story so far,” IEEE Commun. Mag., vol. 36, no. 2, pp. 72-74, Dec. 1998.

[22] J. G. Proakis, Digital Communications, 4th ed. New York: McGrawHill, 2000.

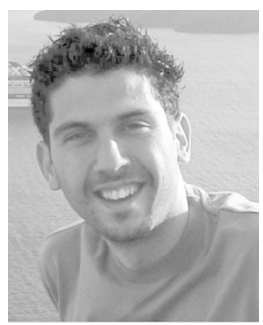

Nestor D. Chatzidiamantis (S'08) was born in Los Angeles, USA, in 1981. He received the Diploma in electrical and computer engineering from the Aristotle University of Thessaloniki, Thessaloniki, Greece, in 2005. Since 2008 he has been working toward the Ph.D. degree at the Department of Electrical and Computer Engineering, Aristotle University of Thessaloniki.

His current research interests include performance analysis over fading channels and free-space optical communications.

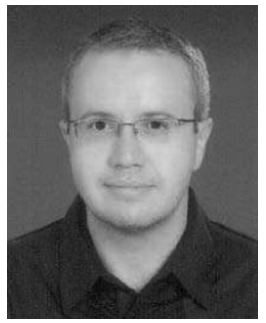

Murat Uysal (SM'07) was born in Istanbul, Turkey, in 1973. He received the B.Sc. and M.Sc. degrees in electronics and communication engineering from Istanbul Technical University, Istanbul, Turkey, in 1995 and 1998, respectively, and the Ph.D. degree in electrical engineering from Texas A\&M University, College Station, in 2001.

Since 2002, he has been with the Department of Electrical and Computer Engineering, University of Waterloo, Waterloo, ON, Canada, where he is currently an Associate Professor. He is currently on sabbatical leave at Özyeğin University, Istanbul, Turkey. His main research interests include communications theory and signal processing for communications with special emphasis on wireless applications. Specific research areas include multiple-input multiple-output (MIMO) techniques, space-time coding, diversity techniques and coding for fading channels, cooperative communications, and free-space optical communications.

Dr. Uysal is an Associate Editor of the IEEE TRANSACTIONS ON WIRELESS COMMUNICATIONS and IEEE COMMUNICATIONS LETTERS, and a Guest Coeditor of the IEEE JOURNAL ON SELECTED AREAS In COMMUNICATIONS' Special Issue on "Optical Wireless Communications." Over the years, he has been a member of the Technical Program Committee of more than 50 international conferences in the communications area. He is a Cochair for the IEEE International Conference on Communications 2007 on Communication Theory Symposium and the Canadian Conference on Electrical and Computer Engineering 2008 on Communications and Networking Symposium. He was a Guest Coeditor for Wiley Journal on Wireless Communications and Mobile Computing's Special Issue on "MIMO Communications" published in 2004.

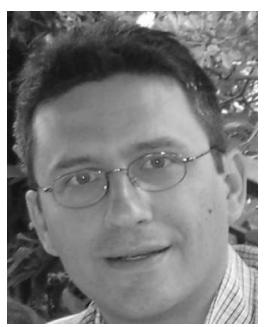

Theodoros A. Tsiftsis (S'02-M'04) was born in Lamia, Greece, in 1970. He received the B.Sc. degree in physics from the Aristotle University of Thessaloniki, Thessaloniki, Greece, in 1993, the M.Sc. degree in digital systems engineering from the Heriot-Watt University, Edinburgh, Scotland, U.K., in 1995, the M.Sc. degree in decision sciences from the Athens University of Economics and Business, Athens, Greece, in 2000, and the Ph.D. degree in electrical engineering from the University of Patras, Patras, Greece, in 2006.

$\mathrm{He}$ is currently an Assistant Professor in the Department of Electrical Engineering, Technological Educational Institute of Lamia, Lamia, Greece. His current research interests include cooperative diversity systems, wireless communications theory, digital communications over fading channels and free-space optical communications.

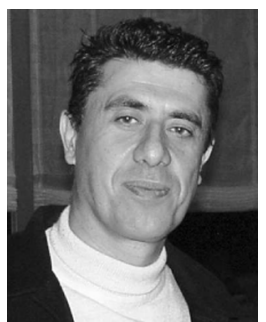

George K. Karagiannidis (M'97-SM'04) was born in Pithagorion, Samos Island. He received the Diploma and Ph.D. degrees in electrical engineering from the University of Patras, Patras, Greece, in 1987 and 1999 , respectively.

From 2000 to 2004, he was a Senior Researcher at the Institute for Space Applications and Remote Sensing, National Observatory of Athens, Greece. In June 2004, he joined Aristotle University of Thessaloniki, Thessaloniki, Greece, where he is currently an Associate Professor of digital communications systems in the Department Electrical and Computer Engineering. He is the author or coauthor of more than 100 technical papers published in scientific journals and presented at international conferences. He is also a coauthor of three chapters in books and author of the Greek edition of a book on Telecommunications Systems. His current research interests include digital communications theory, wireless optical communications, and underwater communications.

Dr. Karagiannidis is a member of the Technical Program Committees for several IEEE conferences, Editorial Boards of the IEEE TRANSACTIONS ON COMMUNICATIONS, IEEE COMMUNICATIONS LETTERS, and Lead Guest Editor of the Special Issue on "Optical Wireless Communications" of the IEEE Journal on SELECTED AREAS IN COMMUNICATIONS. He is a member of the Editorial Board of the European Association for Signal Processing Journal on Wireless Communications and Networking. He is a corecipient of the Best Paper Award of the Wireless Communications Symposium in IEEE International Conference on Communications 2007, Glasgow, U.K., June 2007. 